\title{
H-12. Ophthalmophonogram and its Clinical Application to Carotid-Cavernous Fistula
}

\author{
Hiroyuki TanjI, Kazuo Watanabe, \\ Katsuhiko YamaguchI, Akira SUdA \\ Tsuneji Hika and Shinichiro Endo \\ Department of Surgery, Fukushima Medical College
}

\author{
H-13. The Origin of Positive Brain Scan \\ Autoradiographic Study on the Hypoxic Brain \\ Tatsuya Kobayashi, M. D. \\ Department of Neurosurgery, Nagoya University, School of Medicine \\ Louis BaKay, M. D. \\ Department of Neurosurgery, State University of New York at Buffalo
}

In brain scanning, it is well known that positive scans are often obtained in the cases with non-tumorous intracranial lesion as well as brain tumors. However the causes of the positive scan by such a non-tumorous lesion are still obscure.

The purpose of present paper is to study the mechanisms by which one of molecules commonly used for brain scanning, ${ }^{203} \mathrm{Hg}$-chlormerodrin, and two of extracellular tracers, ${ }^{14} \mathrm{C}$-or ${ }^{3} \mathrm{H}$-inulin, accumulate in the hypoxic rat brain.

Severe acute hypercapnic hypoxia was produced in 12 adult rats by induced hypoventilation for 1 to 5 hours. The mean arterial $\mathrm{PO}_{2}$ was $26.40(18-43) \mathrm{mmHg}$, $\mathrm{PCO}_{2} 104.94(85-123) \mathrm{mmHg}$ and $\mathrm{pH} 7.03(6.13-7.10)$. As a tracer, 50-200 $\mu \mathrm{c}$ of one of the radioactive molecules was injected intravenously 15 minutes to 2 hours before the termination of the experiment.

Structural alterations of the central nervous system were studied by light and electron microscopy. The most severe changes were observed in the neurons of the hippocampus followed by those of the cerebellar and cerebral cortex and basal ganglia. There was significant swelling of the astrocytes. The extracellular space of the white matter showed moderate dilatation.

By observation on the light and electron microscopic autoradiographs, the permeability of the blood-brain barrier increased for both extracellular markers and chlormerodrin. Light microscopic autoradiography revealed that ${ }^{14} \mathrm{C}$ or ${ }^{3} \mathrm{H}$-inulin and ${ }^{14} \mathrm{C}$-sucrose remained in the extracellular space although some sucrose was incorporated in damaged cells. On light and electron microscopic autoradiograms, ${ }^{203} \mathrm{Hg}$-chlormerodrin was seen to be taken by damaged neurons and swollen astrocytes. 
From this study, it might be concluded that not only the increased permeability of blood-brain barrier but also the existence of increaed extracellular space and the selective accumulation of chlormerodrin in damaged cells are the most important factors to make positive scan in anoxic brain lesions.

\title{
H-14. Ultrasonic Diagnosis of Brain Tumors by Extradurally Coherent Compound Scanning Method
}

\author{
Hiroshi Hata, Toshihei Takahashi \\ Hasumiki KaYAma and Yutaka INABA \\ Department of Neurosurgery, Tokyo Medical and Dental University
}

\section{H-15. Clinical Experience and Model Experiment of Cisterno-Lumbar Electro Manometric Queckenstedt Test (G-L EMQ Test)}

\author{
Minoru Thunoda, M. D., Takashi Owada, M. D., \\ Tetsu Kuramae, M. D., Hiroshi Takagi, M. D. \\ and Yoshio Miyasaka, M. D. \\ Depariment of Neurosurgery, Kushiro Rosai Hospital, \\ Kushiro, Hokkaido
}

The Q-Test is a well accepted method to estimate the obstruction of subarachnoid space of the spinal canal. However, we sometimes have been disturbed by the influences of other factors, such as holding of breath, abdominal compression, grunting, sneezing and spasm of the legs during recording the CSF pressure. We have advanced this method up to the C-L EMQ Test, that can avoid the various disturbing factors in clinical recording of Q-Test and estimte the degree of obstruction constantly and quantitatively. We would like to present this method and discuss the quantitative evaluation of the blockage and the meaning of partial blockage upon both clinical experience and medal experiment.

Method: Each of the cisternal and lumbar punctured needles is independently connected to the pressure transducer, which record simultaneously pressure responces of the two points under the managements of gradational jugular compressin using cuff manometer placed around the patient's neck $(20,30,40,50$ and $60 \mathrm{mmHg}$ ), coughing and abdominal compression in flexion, neutral and extension of the neck positionings. If the obstruction be none, the pressure responses between the two points are synchronous and parallel. 